\title{
Review Article \\ Review of the Dynamics of Coalescence and Demulsification by High-Voltage Pulsed Electric Fields
}

\author{
Ye Peng, ${ }^{1,2}$ Tao Liu, ${ }^{1}$ Haifeng Gong, ${ }^{2,3}$ and Xianming Zhang ${ }^{2}$ \\ ${ }^{1}$ School of Mechanical and Electronical Engineering, Lanzhou University of Technology, Lanzhou 730050, China \\ ${ }^{2}$ Engineering Research Centre for Waster Oil Recovery Technology and Equipment, Chongqing 400067, China \\ ${ }^{3}$ School of Mechanical Engineering, Chongqing University of Technology, Chongqing 400054, China \\ Correspondence should be addressed to Ye Peng; ghfpy@cqut.edu.cn
}

Received 30 November 2015; Revised 18 March 2016; Accepted 21 March 2016

Academic Editor: Doraiswami Ramkrishna

Copyright (c) 2016 Ye Peng et al. This is an open access article distributed under the Creative Commons Attribution License, which permits unrestricted use, distribution, and reproduction in any medium, provided the original work is properly cited.

\begin{abstract}
The coalescence of droplets in oil can be implemented rapidly by high-voltage pulse electric field, which is an effective demulsification dehydration technological method. At present, it is widely believed that the main reason of pulse electric field promoting droplets coalescence is the dipole coalescence and oscillation coalescence in pulse electric field, and the optimal coalescence pulse electric field parameters exist. Around the above content, the dynamics of high-voltage pulse electric field promoting the coalescence of emulsified droplets is studied by researchers domestically and abroad. By review, the progress of high-voltage pulse electric field demulsification technology can get a better understanding, which has an effect of throwing a sprat to catch a whale on promoting the industrial application.
\end{abstract}

\section{Introduction}

Oil demulsification dehydration is widely used in petrochemical engineering, resources recycling, metallurgy, equipment manufacturing, and other engineering technologies [1]. The process of demulsification dehydration by a high-voltage pulsed electric field has gained wide attention because of the advantages offered by such a technique, which include low energy consumption, simple structure, and rapid processing speed [2,3]. In 1981, Bailes and Larkai [4] proposed an electric field dehydration scheme for "Pulsed Direct Current (DC) Electric Field + Insulated Electrode," the original mode of pulsed electric field demulsification dehydration. In 1992, Britain Wareham Oil Field adopted induced sedimentation by a pulsed electric field for oil-water separation. The approach can effectively expand the particle size of the droplets from $15 \mu \mathrm{m}$ to $90 \mu \mathrm{m}$ without any chemical demulsifier [5]. Demulsification dehydration by a pulsed electric field consumes only $1 / 18$ and $1 / 29$ of the energy required by $D C$ and alternating current (AC) electric fields, respectively, under the same conditions [6]. High-voltage pulsed electric fields exhibit good performance in demulsification dehydration.
In recent years, demulsification by a pulsed electric field has seen rapid development as a new oil demulsification method. However, research on the dynamic mechanism of demulsification by pulsed electric fields has mostly remained elementary, and the technique still lacks accurate and unified descriptions. The lack of an in-depth understanding of the essential mechanism and inherent laws of demulsification by a pulsed electric field has restricted and hindered the application of this method [7]. Domestic and foreign scholars express a more consistent view of the dynamic mechanism of high-voltage pulsed electric fields promoting the coalescence of emulsified droplets: in water-to-oil emulsified oil, the conductivity of oil and droplets, which may be considered the continuous phase and dispersed phase, respectively, vary considerably $[8,9]$. Droplets are polarised by an external electric field, and the polarised droplets undergo coalescence through collision within a short period of time [10-12]. Dipole coalescence and oscillation coalescence effects are believed to exist in pulsed electric fields, and oscillation coalescence is the main factor influencing droplet coalescence $[2,13]$. To this end, the academic community has conducted 
several studies on the dynamics of pulsed electric fields promoting the coalescence of droplets.

\section{Dipole Coalescence Effect}

Neutral droplets without polarisation charges in oil would be induced dipoles under the influence of electric field induction, and each induced dipole carries equal amounts of opposite charges at both ends [14]. Two induced dipoles could produce interactional electric field forces via the action of dipole moments, and two polarised droplets close to each other can overcome their oil resistance and undergo dipole coalescence [15]. At present, the theoretical models of dipole coalescence include the spherical droplet model and the prolate sphere droplet model.

2.1. Spherical Droplet Model. Studies have suggested that polarised droplets in emulsified oil are arranged along the direction of the electric field. Then, positive and negative ions in droplets move to separate ends and form dipoles; thus, a dipole moment occurs [16]. Two polarised droplets are equivalent to two dipoles located in the geometric centre of the droplet, as shown in Figure 1. Assuming that the dipole moments of two polarised droplets were $\mu_{3}$ and $\mu_{4}$, respectively, the field energy between dipoles could be obtained by the energy method [17]:

$$
\begin{aligned}
& W=-\frac{\mu_{3} \mu_{4}}{4 \pi \varepsilon_{0} \varepsilon_{2} r^{3}}\left(3 \cos ^{2} \theta-1\right), \\
& \mu_{3}=4 \pi r_{1}^{3} \varepsilon_{0} \varepsilon_{2} E\left(\frac{\varepsilon_{1}-\varepsilon_{2}}{\varepsilon_{1}+2 \varepsilon_{2}}\right), \mu_{4}=4 \pi r_{2}^{3} \varepsilon_{0} \varepsilon_{2} E\left(\frac{\varepsilon_{1}-\varepsilon_{2}}{\varepsilon_{1}+2 \varepsilon_{2}}\right),
\end{aligned}
$$

where $r_{1}$ and $r_{2}$ are the radii of two droplets, $\varepsilon_{0}$ is the vacuum dielectric constants, $\varepsilon_{1}$ and $\varepsilon_{2}$ are the relative dielectric constants of the droplet and the oil, $E$ is the electric field intensity, and $W$ is the field energy between two dipoles.

The partial derivative of $W$ with respect to $r$ can be obtained. Considering that $\varepsilon_{1} \gg \varepsilon_{2}$, the radial electric field force between the two droplets could be calculated as

$$
F_{r}=-\left.\frac{\partial W}{\partial r}\right|_{r=d}=-\frac{6 \pi \varepsilon_{0} \varepsilon_{2} r_{1}^{3} r_{2}^{3} E^{2}}{r^{4}}(3 \cos 2 \theta+1),
$$

where $d$ is the distance between two drops.

Polarisation is a major influence of an external electric field on droplets in oil. Thus, the magnitude and direction of the electric field force between the two droplets change with changing droplet centre lines. In (2), the electric field force between the two polarised droplets involves attractive force; the relation is as follows:

$$
3 \cos 2 \theta+1>0
$$

It can be obtained that

$$
\theta<54.7^{\circ} \text { or } \theta>125.3^{\circ} \text {. }
$$

Williams [18] determined that the radial force between two droplets is repulsive when $\theta$ is located between $54.7^{\circ}$ and

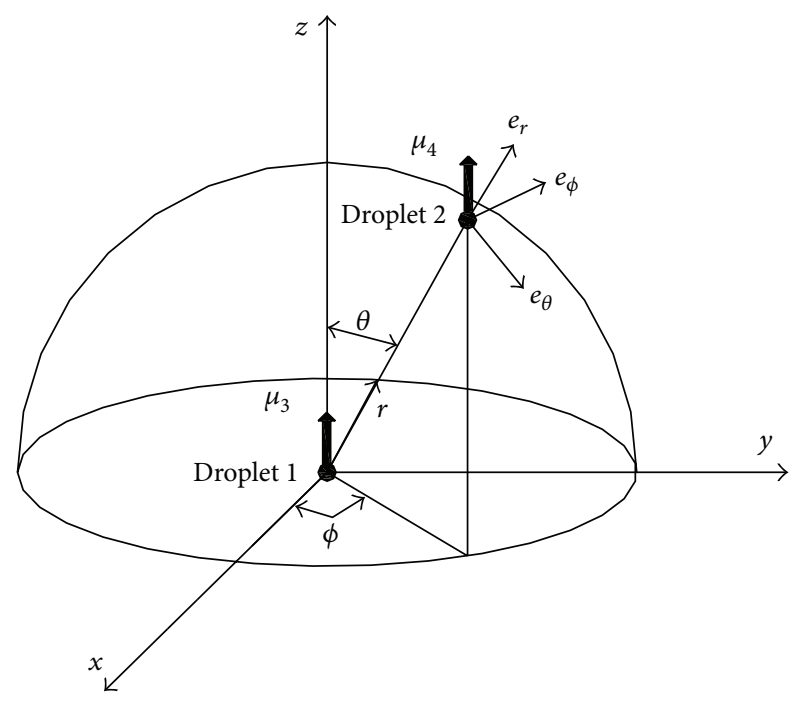

FIGURE 1: In sphere coordinates, two droplets were polarized and become dipoles [17]. $\mu_{3}$ and $\mu_{4}$ were the dipole moments of droplet 1 and droplet 2 , respectively. $e_{r}, e_{\phi}$, and $e_{\theta}$ were the unit vectors.

$125.3^{\circ}$. Eow and Ghadiri [19] confirmed the above conclusion by experiments.

Bailey, Bailes, and Waterman et al. [20-22] researched the electric field force of two spherical polarised droplets possessing the same radii in the case where the line of centres was parallel to the direction of the electric field and found that

$$
F=-\frac{24 \pi \varepsilon_{0} \varepsilon_{2} R_{0}^{6} E^{2}}{\left(d+2 R_{0}\right)^{4}}
$$

where $R_{0}$ is the radius of the droplet.

It can be considered that the above electric field force is the primary factor influencing droplet coalescence. It is noted that when the droplet spacing is smaller than a certain critical distance, the above expression is no longer applicable [23]. In addition, the spherical droplet model does not take into account the deformation of droplets in the electric field.

2.2. Prolate Sphere Droplet Model. Under the effect of the electric field, droplets in oil produce polarisation charges at both ends, which are of opposite polarity to the electrode plates. The droplets are stretched to approximately prolate spheres under the action of an electric field force [15]. As shown in Figure 2, Gong et al. [24] calculated the polarised electric field force of two approximate prolate sphere droplets in an arbitrary space by using the Maxwell stress tensor method. The radial electric field force $F_{r}$ and the tangential electric field force $F_{\alpha}$ between the two prolate sphere droplets could be obtained as

$$
\left(\begin{array}{l}
F_{r} \\
F_{\alpha}
\end{array}\right)=\varepsilon_{2} \varepsilon_{0} b_{2}^{2} \lambda^{2} E^{2} \cdot\left[\begin{array}{cc}
\cos \alpha^{\prime} & \sin \alpha^{\prime} \\
-\sin \alpha^{\prime} & \cos \alpha^{\prime}
\end{array}\right]\left(\begin{array}{l}
K_{1} \\
K_{2}
\end{array}\right),
$$

where $b_{2}$ is the minor semiaxis of droplet $2, \lambda$ is the stretching ratio of droplet, $K_{1}$ and $K_{2}$ are coefficients of force, and $\alpha^{\prime}$ is the electric field angle. 


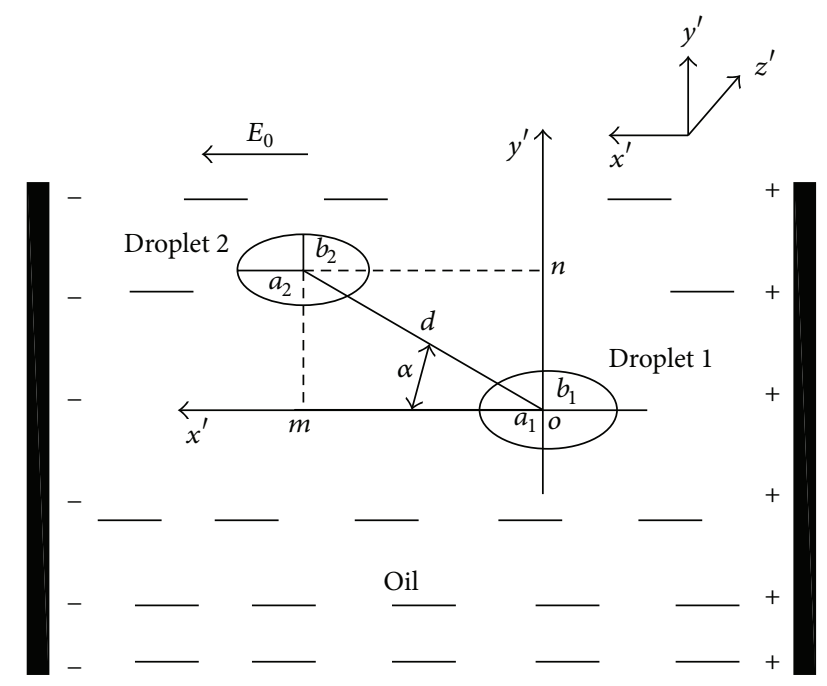

FIGURE 2: Model of two ellipsoidal deforming droplets [24]. $a_{1}$ and $b_{1}$ and $a_{2}$ and $b_{2}$ were the major and minor semiaxis of droplet 1 and droplet 2, respectively. The distance of two droplets' center was $d$ and the electric field angle was $\alpha$.

Similarly, the critical angle of two droplets at which attraction-repulsion occurs can be obtained as follows:

$$
\alpha_{\text {crit }}^{\prime}=\arctan \left(-\frac{K_{1}}{K_{2}}\right) .
$$

Considering the influence of droplet deformation on the critical angle, the following modifier formula is established:

$$
\alpha_{\text {crit }}=\arctan \left(\frac{\tan \alpha_{\text {crit }}^{\prime}}{\lambda}\right),
$$

where $\alpha_{\text {crit }}$ is the correction value of critical angle.

2.3. Droplet Dipole Coalescence. Droplets in the electric field come close to each other under the action of polarisation force. Then, the oil between the two droplets is exhausted. Charles and Mason [25], Allan and Mason [26], and Brown and Hanson [27] found that the oil film immediately bursts when the spacing between two droplets approaches the critical value, and then dipole coalescence occurs. With the asymptotic laws, Atten et al. [28] suggested that the critical separation of (nondeformed) droplets is a function of the applied field $E$, shown in (9). Equation (9) might be used as coalescence criteria in attempts to simulate numerically the evolution of water-in-oil emulsions subjected to an electric field

$$
\left(\frac{s_{0}}{R_{0}}\right)_{\text {crit }} \cong 8\left[E \sqrt{\frac{\varepsilon_{2} R_{0}}{\gamma}}\right]^{1.22},
$$

where $s_{0}$ is the thickness of oil film between droplets and $\gamma$ is the interfacial tension. When the droplet spacing is smaller than the critical value, the oil between droplets is rapidly exhausted by the electric field force. The electric field intensity importantly contributes to oil film burst, and the coalescence angle amongst the droplets and contact time decrease with increasing the electric field intensity until instantaneous coalescence occurs. Allan and Mason [29] suggested that when two droplets come into contact with each other in the AC/DC electric field, spark discharges pierce the oil film, causing droplet coalescence. Harpur et al. [30] held the same perspective and suggested that the droplets are close to each other because of turbulent mixing because the polarised electric field gravitation amongst droplets is limited.

\section{Oscillation Coalescence Effect}

Taylor [31] suggested that droplets in an electric field form induced dipoles, and both ends of each dipole produced two tensions, which were equal but in opposite direction. Under tension, droplets are stretched into prolate spheres and return to their original shape after the electric field is removed. Zhang et al. and Torza et al. [7, 32] suggested that, under the effect of a pulsed DC electric field, the repeated stretching deformation of droplets represents a type of periodic vibration. Vibrations could increase the probability of collision and enhance the collision kinetic energy, thereby weakening the mechanical strength of the interfacial film. Droplet vibration is the main cause of oscillation coalescence, and pulsed electric field intensity and frequency are two important factors influencing droplet vibration [33].

3.1. Influence of Pulse Frequency on Oscillation Coalescence. Pulsed electric field frequency exerts an important impact on the coalescence of droplets in emulsified oil. Brown and Hanson [27] found that each emulsified oil system exhibits an optimal pulsed electric field frequency that can enhance droplet vibration and promote oil film burst and droplet coalescence. Charles and Mason [34] proposed a transition effect theory and suggested that the electric field induces charges on the droplet and the small droplets begin to disperse with the increase of electric field intensity, but droplets have a quick coalescence by the instantaneous pulsed electric field, and they found that the optimal frequency is beneficial to droplet coalescence. The dielectric relaxation theory of Bailes and Larkai [35] also suggests that when the pulse frequency is at the optimal demulsification frequency, droplets could be completely polarised at the peak of the electric field. At this moment, the electric field force between droplets is the largest, considerably improving the probability of droplet collision, and the effect of droplets coalescence is significant. Williams [36] suggested that droplets in an electric field vibrate along with the pulse frequency. According to the theory of vibration dynamics, the vibration amplitude increases with the increasing electric field intensity and the vibration amplitude depends on the electric field frequency. When the electric field is at a certain frequency band and the vibration of droplets is the most intense and the most beneficial to droplet coalescence, this frequency is the optimal coalescence frequency.

For the package insulated electrode, the optimal pulsed electric field frequency that promotes droplet coalescence is 
related to the electric relaxation time of the electrode package layer according to Maxwell-Weiner effect theory [37]

$$
f_{p}=\frac{1}{2 \pi \tau_{M-W}}, \quad \tau_{M-W}=\frac{\left(d_{s} \varepsilon_{2}+d_{c} \varepsilon_{s}\right) \varepsilon_{0}}{d_{s} C_{c}+d_{c} C_{s}},
$$

where $f_{p}$ is the optimal demulsification frequency, $\tau_{M-W}$ is electric relaxation time of encapsulation layer, $d_{s}$ and $d_{c}$ are the thickness of the package layer and the oil, $\varepsilon_{s}$ is the relative dielectric constants of the package layer, and $C_{c}$ and $C_{s}$ are the conductivity of the package layer and the oil. Zhang et al. [7] suggested that droplets resonate when the electric field frequency approaches or is equal to the free vibration frequency of droplets in emulsified oil, and the optimal pulsed electric field coalescence frequency could be obtained using linear vibration theory:

$$
\omega_{0}=\left(\frac{4 C_{R} \gamma}{\left(1+C_{m}\right) R^{3} \rho}\right)^{0.5},
$$

where $\omega_{0}$ is the free vibration frequency of droplets, $C_{m}$ is the additional quality coefficient, and $C_{R}$ is the restoring force coefficient.

Gong et al. [38] found that the vibration of droplets under the action of a pulsed electric field force resembles singledegree, nonlinear parametric excitation vibration and that the main impetus of this vibration originates from the harmonic component of electric field force. Thus, a dynamic model of droplet vibration could be established as follows:

$$
\frac{d^{2} \chi}{d t^{2}}+A \varphi(\chi) \frac{d \chi}{d t}+B f(\chi)=G q(t) e(\chi),
$$

where $A, B$, and $G$ are the coefficients of stress term, $\chi$ is the vibration amplitude of droplets, and $\varphi(\chi), f(\chi)$, and $e(\chi)$ are the functions of stress term. The team also suggested that the optimal pulsed electric field coalescence frequency deviates from the free vibration frequency of droplets because of oil viscosity. Greater viscosity leads to larger deviations. The optimal coalescence frequency may not exist if the viscosity is too great, as confirmed in Eow and Ghadiri's [15] experiments.

Numerous studies on optimal demulsification frequency have been conducted. Wang [39] suggested that the forming and disappearing time of electric short-circuit were different for the emulsion, being acted on by the electric field, with different water contents and the optimal demulsification frequency allows the pulse output time to be shorter than the short-circuit forming time, and so the accident of shortcircuit would not happen with the frequency. Liu and Li [8] suggested that insulated electrodes could be equivalent to the $\mathrm{RC}$ circuit and that the effect of demulsification is reduced with the increasing electric field frequency mainly because of the voltage decrease in the emulsion. This finding illustrates the presence of the optimal demulsification frequency in the pulsed electric field. Wang [40] suggested that the electric field force is related to electric field frequency. At extremely low frequencies, the distribution voltage of emulsion droplets is small because of the large capacitance; thus, a lower electric field intensity, which is not conducive to droplet coalescence, is obtained. When the frequency is excessively large, the droplets cannot properly respond to changes in the external electric field. Moreover, the charge of the induced electric charges is relatively small, resulting in a smaller electric field force that is also not conducive to droplet coalescence. These findings illustrate the existence of the optimal demulsification frequency in a pulsed electric field. Yang et al. [41] use the double capacitance model to estimate the optimal frequency of promoting droplet coalescence in pulsed electric fields produced by cylindrical electrodes.

Peng et al. [42] suggested that an optimal pulse frequency of droplet coalescence exists in the initial emulsified oil system. Droplet size increases gradually in the process of coalescence and the optimal coalescence frequency changes. In addition, when using advanced pulsed electric fields with variable frequency to promote the coalescence of droplets in emulsion oil, the method for determining the variable frequency and the time interval may be given by

$$
\begin{aligned}
\omega_{\text {reso }} & =\Psi\left(\omega_{0}, m, n\right), \\
t_{1} & =\frac{8}{15} \frac{\mu}{\varepsilon_{2} \varepsilon_{0} E^{2}}\left[\left(\frac{\pi}{6 \phi}\right)^{5 / 3}-1\right],
\end{aligned}
$$

where $\omega_{\text {reso }}$ is the parametric excitation resonance frequency of the droplet, $m$ is equal to $\left(\varepsilon_{0} \varepsilon_{2} E^{2} R\right) / \gamma, n$ is $\left(\varepsilon_{0} \varepsilon_{2} E^{2} R \sqrt{\rho}\right) /(\mu \sqrt{8 \gamma}), \mu$ is the viscosity of oil, $\gamma$ is the interfacial tension stress, $R$ is the radius of the droplet, $\phi$ is the droplets volume fraction in oil, and $t_{1}$ is the time interval of electric field changing. Experimental results [43] also show that the pulsed electric field with variable frequency exhibits superior effects in comparison with those of pulsed electric fields with a constant frequency on the coalescence of emulsion droplets.

\subsection{Influence of Electric Field Intensity on Oscillation Coales-} cence. In addition to pulsed electric field frequency, another important parameter affecting oscillation coalescence is pulsed electric field intensity. Through experiments, Taylor [47] suggested that the maximum coalescence of droplets in emulsified oil is related to the intensity of the external electric field to a certain extent. Induced droplets with charges would burst when the electric field intensity exceeds a certain value, and the effect of coalescence decreases significantly. Gan [48] found that droplets undergo tensile deformation under the action of a pulsed electric field with higher amplitude. One end of the droplet became ellipsoid in shape, whereas the other end resembled a cusp. Greater electric field intensities result in larger deformations of the droplets. Through experiments, Eow and Ghadiri [19] observed that a droplet under a pulsed electric field gradually stretches with increasing electric field intensity. The droplet then breaks down into two small droplets, consistent with the research of Taylor [49]. The tandem thus theoretically defined the dimensionless parameter of electric field We (Weber number $=2 R \varepsilon_{0} \varepsilon_{2} E_{0}^{2} / \lambda$ ), which indicates the significance of the electrostatic stress with respect to the interfacial tension and similar to that in fluid mechanics, to measure the stability of droplets and define the 


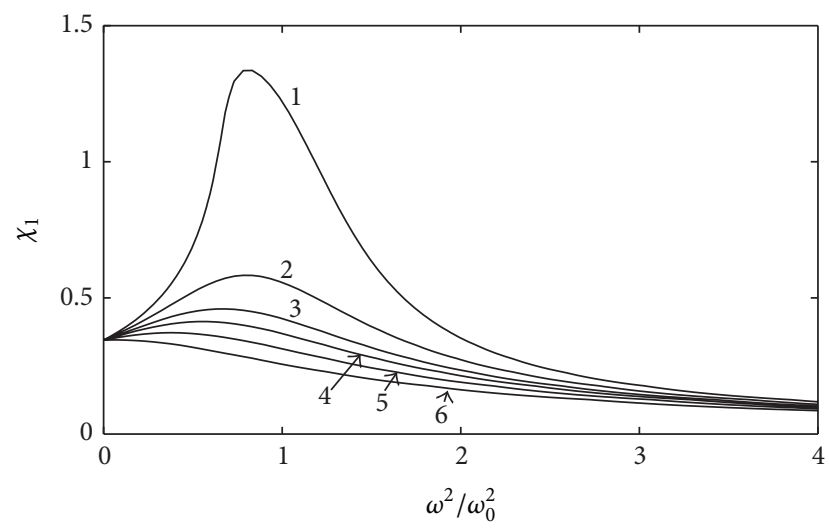

FIGURE 3: Curve of amplitude-frequency of droplet under different oil's viscosity [44]. Curve 1-curve 6 were curves of amplitudefrequency characteristic of the droplet in oils whose viscosities were $10,30,40,47.2,55$, and $70 \mathrm{~Pa} \cdot \mathrm{s}$, respectively. It showed that the smaller the oil viscosity, the larger the vibration amplitude.

critical ratio of the major semiaxes to the minor semiaxes when the droplet was burst. Evidently, an optimal intensity of pulsed electric field also exists for the oscillation coalescence of droplets.

Bailes and Dowling [33] considered that the square wave could produce the maximum electric field intensity after comparing the pulse square wave, the pulse half-wave, and the pulse triangle wave. Nishiwaki et al. [50] estimated the optimal electric field intensity of droplet coalescence

$$
E_{c}=0.64 \sqrt{\frac{\lambda}{2 \varepsilon_{2} \varepsilon_{0} R_{0}}} .
$$

The electric field force of droplet deformation was determined by the amplitude of pulsed electric field intensity, which exerts an influence on the parametric excitation vibration of droplets in oil. Higher electric field intensities result in greater resonance amplitudes of the droplets, but the change in the pulsed electric field intensity cannot determine the monotonic change in coalescence frequency [51].

\subsection{Influence of System Physical Parameters on Oscillation} Coalescence. At present, research on emulsified droplet oscillation coalescence mainly focuses on the calculation and optimisation of the electric field frequency and intensity. However, the physical parameters of the medium in an emulsified oil system, including the oil viscosity, interfacial tension stress, and the oil dielectric constant, also exert important impacts on droplet vibration.

Through the establishment of the vibration kinetic model of a single droplet in oil, Gong and Peng [44] found that the viscous force of oil could hinder droplet stretching deformation. The demulsification optimal electric field frequency and resonant amplitude decrease with the increasing oil viscosity, as shown in Figure 3. By using the amplitude-frequency characteristics of the stretching vibration of droplets, the team theoretically explained the impact of oil viscosity on the optimal demulsification frequency, as well as the causes of the

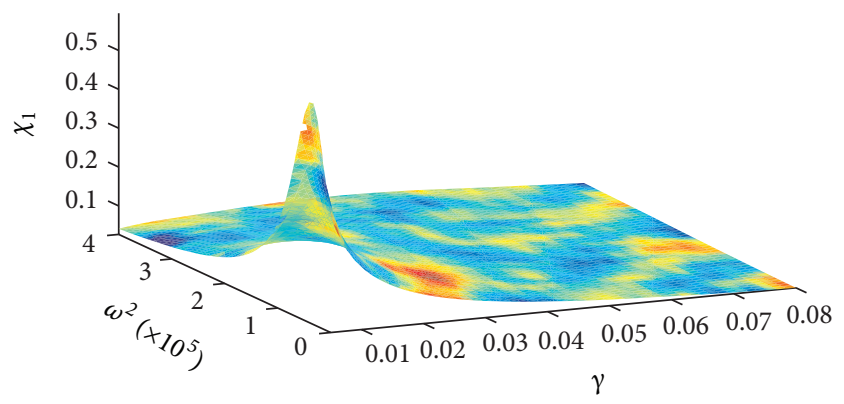

FIGURE 4: Influence relation graph of interface tensile stress on amplitude [45]. The unstable vibration could be inhibited by increasing the electric field frequency. The vibration amplitude of droplets suddenly increased with decreasing the interface tensile stress.

optimal demulsification frequency deviating from the natural vibration frequency.

The group further studied the influence of the interface tensile stress of oil droplets on the vibration of these droplets in a pulsed electric field [45] and drew the following conclusion: the vibration amplitude peak increases with decreasing interface tensile stress. Under a small interface tensile stress, the droplets undergo unstable vibration. This vibration could be bettered by increasing the electric field frequency, as shown in Figure 4. The resonance frequency of droplets increases with increasing interface tensile stress. Therefore, droplets of large interfacial tension stress show good demulsification under a high-frequency electric field.

The oil dielectric constant has a direct influence on the force acting on droplet by electric field. The oil, with high dielectric constant, has a better performance of electrical conductivity and the droplet is polarized easily, which promotes larger electroforce acting on droplet [52]. In pulsed electric field, the high dielectric constant of oil makes the vibration amplitude of droplets increase although it does not have a direct correlation to the coalescence frequency of droplets. In addition, the radius of droplet affects directly the frequency of vibration. The smaller the droplet radius is, the greater the droplet natural frequency is [7]. So, the coalescence frequency of droplets with the smaller radius has a tendency of increasing.

\section{Matching of Electric Field Parameters}

The contributions of pulsed electric field parameters to the coalescence of droplets in oil are not identical: the pulsed electric field intensity directly affects the tensile deformation of droplets, the pulsed electric field frequency exerts a larger influence on the vibration of droplets, and the pulse duty ratio exerts an important influence on the polarisation of the droplet surface charge and the control of short-circuiting of droplet chains between electrode plates. Viewed from the perspective of effective energy, the contributions of pulsed electric field waveforms, such as the unidirectional square wave, the sine wave, and the triangle wave, also vary in promoting the coalescence of droplets [53]. Therefore, 


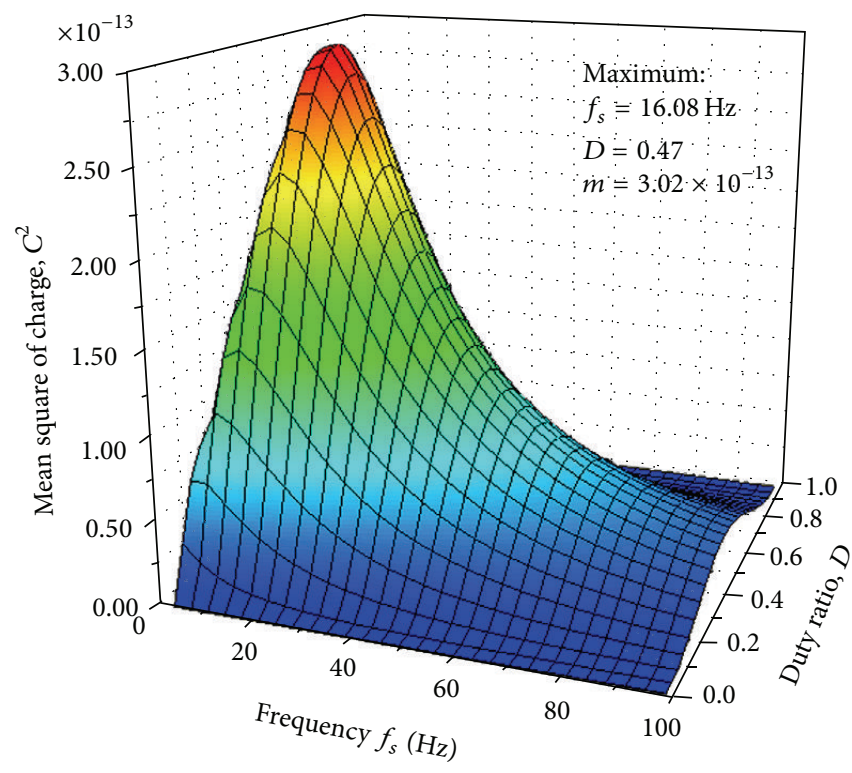

(a)

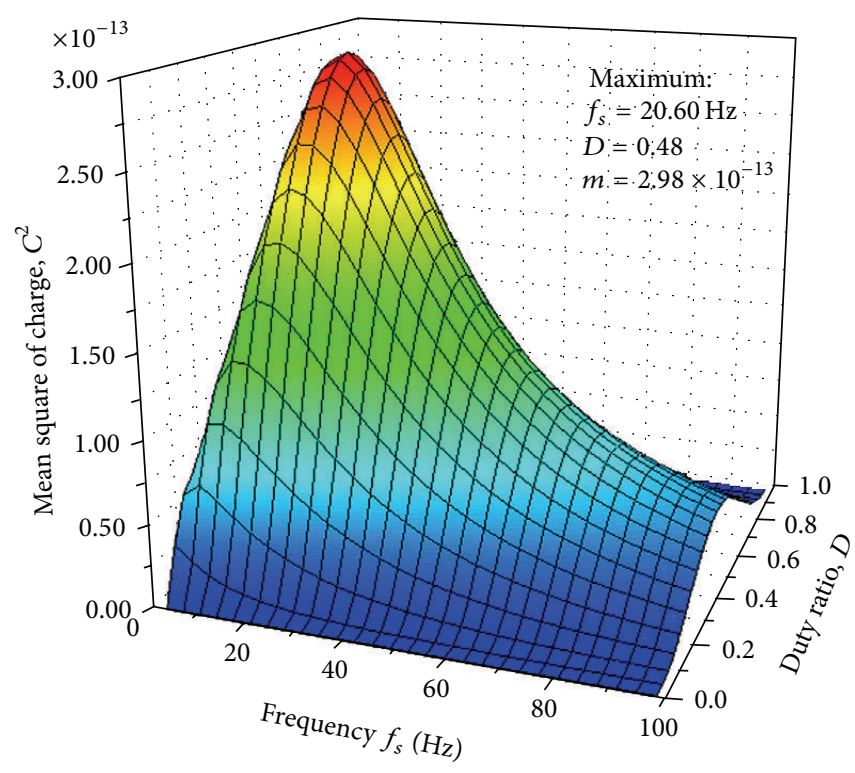

(b)

FIGURE 5: Quantity of electric charge as a function of frequency and duty ratio at water contents $10 \%$ and $30 \%$ [41]. Obviously, there are the matching relations of frequency and duty ratio with the optimal coalescence of droplets.

matching of electric field parameters is an important subject in the dynamic research of pulsed electric fields promoting coalescence and presents important guiding significance in the industrial applications of pulsed electric field demulsification technology.

Bailes and Dowling [33] suggested that the coalescence efficiency of droplets in the pulsed electric field is a function of the electric field amplitude, the electric field frequency, and the pulse waveform. These parameters presented optimal values and strong matching relationship. According to theory of charge relaxation, Midtgård [54] found that the promotion of coalescence by a pulsed electric field is a function of the electric field frequency and pulse duty ratio and that the optimal coalescence electric field frequency and optimal coalescence pulse duty ratio could be obtained by optimising the parameters of coalescence electric field. Yang et al. [41] obtained matching functions of the polarised electric field as well as the parameters of the pulsed electric field; the optimal coalescence electric field frequency and the optimal coalescence pulse duty ratio of the water content were $10 \%$ and 30\%, respectively, as shown in Figure 5.

Gong et al. [46] suggested that the stretching ratio, $\lambda$, of droplets in oil cannot indefinitely increase with increasing electric field intensity. By taking $\lambda \leq 1.9$ as the control condition of stable droplet deformation, the group established a calculation graph of optimal demulsification electric field parameters shown in Figure 6. The optimal matching values of the coalescence electric field frequency and electric field intensity of droplets in SsangYong $70 \mathrm{SN}$ base oil could be obtained by using Figure 6 . Under the given oil viscosity $\mu$, interfacial tension stress $\gamma$, medium dielectric constant $\varepsilon_{2}$, droplets density $\rho$ and droplets initial radius $R, t$, shown in Figure 6, was 2.765 by the relation of $t=3.4152 \mu^{2} /(R \rho \gamma)$.

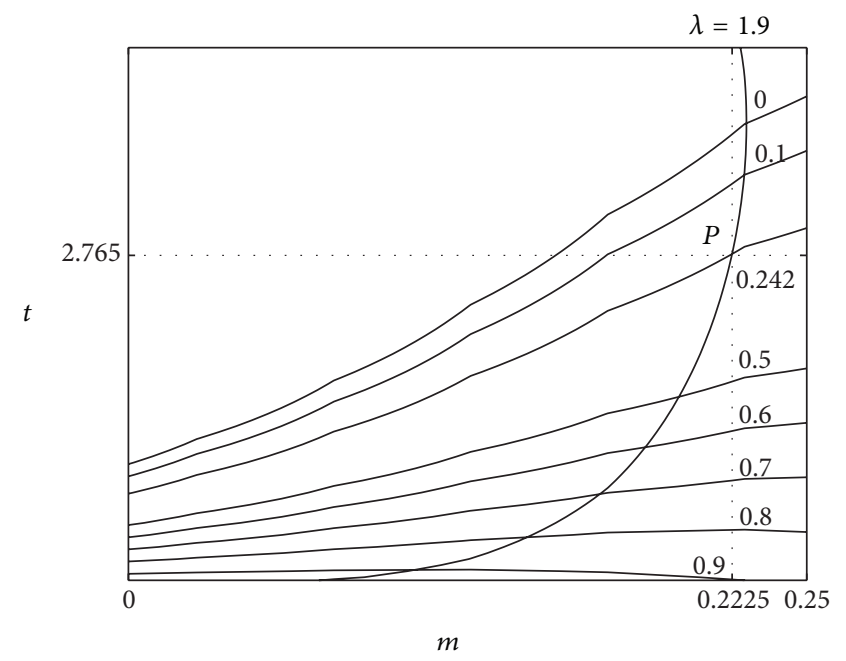

FIGURE 6: Sketch map of confirming optimal demulsification electric field parameters [46]. Using the map, the frequency and intensity of optimal coalescence could be obtained by $t$ and $m$.

The point $P$ was the intersection of curve $t=2.765$ and curve $\lambda=1.9$, and curve $\omega^{2} / \omega_{0}^{2}=0.242$ also went through the point $P$ by which the optimal coalescence frequency could be calculated. And a vertical, going through $P$, intersected the horizontal axis of coordinate system and $m=0.2225$ was obtained. By the relation of $m=\varepsilon_{0} \varepsilon_{2} E^{2} R / \gamma$, the optimal coalescence intensity $E$ could be calculated. This figure is of important guiding significance in the parameter selection and structural design of high-performance demulsification devices featuring pulsed electric fields. 


\section{Conclusion}

As an effective physical demulsification method, application of high-voltage pulsed electric field is widely used to dehydrate emulsified oil. Researchers have summarised the dynamic behaviour of pulsed electric fields promoting coalescence as dipole coalescence and oscillation coalescence, and number of studies have been conducted regarding these two effects. In early dynamic studies of dipole coalescence, researchers commonly used the spherical dipole model to study the forces between droplets. Considering the deformation of droplets in oil subjected to an electric field, researchers have studied the action between polarised droplets in an arbitrary space by the double prolate sphere droplet model. The study of oscillation coalescence has mainly concentrated on solving the optimal coalescence frequency. However, considering only the optimal demulsification frequency is insufficient. The influences of pulsed electric field intensity, pulse duty ratio, and physical parameters of the system must also be considered. The matching of the optimal demulsification parameters presents good guiding significance in the practical applications of pulsed electric fields. The efficiency of demulsification with a single pulsed electric field is relatively limited. Coupling physical fields, such as the centrifugal field and the temperature field of negative pressure, will be the developmental mainstream of this technology. Therefore, dynamic research on coupled fields promoting coalescence demulsification may be expected to be a popular topic in the future.

\section{Competing Interests}

The authors declare that they have no competing interests.

\section{Acknowledgments}

This work was partially supported by grants from the Chinese National Natural Science Foundation (Grant no. 21206204), Projects of CQ CSTC (Grant nos. cstc2015jcyjA90018 and cstc2014yykfB90002), and the Chinese Postdoctoral Science Foundation (Grant no. 2015M572640XB).

\section{References}

[1] R. Y. Chang, Aviation Oil Dehydration, Research Department of Air Force Oil, Beijing, China, 1986.

[2] J. S. Eow and M. Ghadiri, "Electrostatic enhancement of coalescence of water droplets in oil: a review of the technology," Chemical Engineering Journal, vol. 85, no. 2-3, pp. 357-368, 2002.

[3] X. Qi and Y.Zhang, "Study on condition of breakdown emulsion by pulse electric field," Membrane Science and Technology, vol. 13, pp. 51-55, 1993.

[4] P. J. Bailes and S. K. L. Larkai, "An experimental investigation into the use of high voltage DC fields for liquid phase separation," Transactions of the Institution of Chemical Engineers, vol. 59, pp. 229-237, 1981.

[5] J. Zhang, "High voltage pulsed DC electric field effect mechanism of demulsification of crude oil emulsion," Oil and Gas Field Surface Engineering, vol. 23, pp. 13-15, 2004.
[6] W. J. Zhou, "Experimental and mechanism study on the dehydration of high voltage electric pulse oil," High Voltage Technology, vol. 2, pp. 24-26, 1995.

[7] J. Zhang, S. Dong, and Q. Gan, "Dynamic model of liquid droplets of water-in-oil emulsions with high-frequency pulsating electrical field," Journal of Chemical Industry and Engineering, vol. 58, no. 4, pp. 875-880, 2007.

[8] B. J. Liu and S. Y. Li, "Study on the mechanism of W/O type emulsion breaking by high voltage pulsed electric field," Membrane Science and Technology, vol. 15, pp. 49-56, 1995.

[9] J. Q. Feng, "Dielectrophoresis of a deformable fluid particle in a nonuniform electric field," Physical Review E, vol. 54, no. 4, pp. 4438-4449, 1996.

[10] N. Aske, H. Kallevik, and J. Sjöblom, "Water-in-crude oil emulsion stability studied by critical electric field measurements. Correlation to physico-chemical parameters and near-infrared spectroscopy," Journal of Petroleum Science and Engineering, vol. 36, no. 1-2, pp. 1-17, 2002.

[11] W. He, J. S. Chang, and M. H. I. Baird, "Formation and dispersion of small water droplets in an insulating oil under a pulsed electric field," in Proceedings of the IEEE Annual Report of the Conference on Electrical Insulation and Dielectric Phenomena, vol. 1, pp. 361-364, Millbrae, Calif, USA, October 1996.

[12] Ø. Midttun, H. Kallevik, J. Sjöblom, and O. M. Kvalheim, "Multivariate screening analysis of water-in-oil emulsions in high external electric fields as studied by means of dielectric time domain spectroscopy. III. Model emulsions containing asphaltenes and resins," Journal of Colloid and Interface Science, vol. 227, no. 2, pp. 262-271, 2000.

[13] T. Shimomura and K. Ito, "Frequency domain electric birefringence study of water-in-oil microemulsion droplets," Colloids and Surfaces A: Physicochemical and Engineering Aspects, vol. 209, no. 2-3, pp. 281-287, 2002.

[14] R. Ceolho and J. L. Lv, Dielectric Physics, Science Press, Beijing, China, 1984.

[15] J. S. Eow and M. Ghadiri, "Motion, deformation and break-up of aqueous drops in oils under high electric field strengths," Chemical Engineering and Processing, vol. 42, no. 4, pp. 259-272, 2003.

[16] H. F. Gong, Y. G. Shi, and L. Mei, "Polarization model and calculation of W/O type emulsion droplet under electric field," Journal of Logistics Engineering College, vol. 25, pp. 44-47, 2009.

[17] C. Z. Ni, "Two dipole interaction energy and interaction force," Journal of Science of Teachers, vol. 23, pp. 30-33, 2003.

[18] T. J. Williams, The resolution of water-in-oil emulsion by the application of an external field [Ph.D. thesis], University of Southampton, 1989.

[19] J. S. Eow and M. Ghadiri, "Drop-drop coalescence in an electric field: the effects of applied electric field and electrode geometry," Colloids and Surfaces A: Physicochemical and Engineering Aspects, vol. 219, no. 1-3, pp. 253-279, 2003.

[20] T. J. Williams and A. G. Bailey, "Changes in the size distribution of a water-in-oil emulsion due to electric field induced coalescence," IEEE Transactions on Industry Applications, vol. 22, no. 3, pp. 536-541, 1986.

[21] P. J. Bailes and E. H. Stitt, "Column liquid contacting with vigorous agitation balanced by electrostatic coalescence," Chemical Engineering Research and Design, vol. 65, pp. 514-523, 1987.

[22] L. C. Waterman, "Electrical coalescers," Chemical Engineering Progress, vol. 61, pp. 51-57, 1965. 
[23] X. Zhang, O. A. Basaran, and R. M. Wham, "Theoretical prediction of electric field-enhanced coalescence of spherical drops," AIChE Journal, vol. 41, no. 7, pp. 1629-1639, 1995.

[24] H. F. Gong, S. Y. Song, and Y. Peng, "Theoretical calculations of electrostatic force between ellipsoidal drops in W/O emulsion," CIESC Journal, vol. 60, no. 7, pp. 1719-1724, 2009.

[25] G. E. Charles and S. G. Mason, "The mechanism of partial coalescence of liquid drops at liquid/liquid interfaces," Journal of Colloid Science, vol. 15, no. 2, pp. 105-122, 1960.

[26] R. S. Allan and S. G. Mason, "Effects of electric fields on coalescence in liquid+liquid systems," Transactions of the Faraday Society, vol. 57, pp. 2027-2040, 1961.

[27] A. H. Brown and C. Hanson, "Effect of oscillating electric fields on coalescence in liquid+liquid systems," Transactions of the Faraday Society, vol. 61, pp. 1754-1760, 1965.

[28] P. Atten, L. Lundgaard, and G. Berg, "A simplified model of electrocoalescence of two close water droplets in oil," Journal of Electrostatics, vol. 64, no. 7-9, pp. 550-554, 2006.

[29] R. S. Allan and S. G. Mason, "Particle motions in sheared suspensions. XIV. Coalescence of liquid drops in electric and shear fields," Journal of Colloid Science, vol. 17, no. 4, pp. 383408, 1962.

[30] I. G. Harpur, N. J. Wayth, A. G. Bailey, M. T. Thew, T. J. Williams, and O. Urdahl, "Destabilisation of water-in-oil emulsions under the influence of an A.C. electric field: experimental assessment of performance," Journal of Electrostatics, vol. 40-41, pp. 135-140, 1997.

[31] S. E. Taylor, "Investigations into the electrical and coalescence behaviour of water-in-crude oil emulsions in high voltage gradients," Colloids and Surfaces, vol. 29, no. 1, pp. 29-51, 1988.

[32] S. Torza, R. G. Cox, and S. G. Mason, "Electrohydrodynamic deformation and burst of liquid drops," Philosophical Transactions of the Royal Society of London A, vol. 269, no. 1198, pp. 295310, 1971.

[33] P. J. Bailes and P. D. Dowling, "The production of pulsed E.H.T. voltages for electrostatic coalescence," Journal of Electrostatics, vol. 17, no. 3, pp. 321-328, 1985.

[34] G. E. Charles and S. G. Mason, "The coalescence of liquid drops with flat liquid/liquid interfaces," Journal of Colloid Science, vol. 15, no. 3, pp. 236-267, 1960

[35] P. J. Bailes and S. K. L. Larkai, "Liquid phase separation in pulsed D. C. fields," Transactions of the Institution of Chemical Engineers, vol. 60, no. 2, pp. 115-121, 1982.

[36] T. J. Williams, Private communication, 2002.

[37] P. J. Bailes, "Electrically augmented settlers and coalescers for solvent extraction," Hydrometallurgy, vol. 30, no. 1-3, pp. 417430, 1992.

[38] H. F. Gong, Y. Peng, H. H. Shang, Z. Yang, and X. Zhang, "Nonlinear vibration of a water drop subjected to high-voltage pulsed electric field in oil: estimation of stretching deformation and resonance frequency," Chemical Engineering Science, vol. 128, pp. 21-27, 2015.

[39] F. C. Wang, "Study on the technology of the electric dehydration of the compound drive," Oil and Gas Field Surface Engineering, vol. 21, pp. 57-58, 2002.

[40] L. C. Wang, "Electrical demulsification mechanism," Petroleum Refining, vol. 7, pp. 47-53, 1992.

[41] D. H. Yang, M. H. Xu, L. M. He et al., "The influence and optimisation of electrical parameters for enhanced coalescence under pulsed DC electric field in a cylindrical electrostatic coalescer," Chemical Engineering Science, vol. 138, pp. 71-85, 2015.
[42] Y. Peng, T. Liu, H. Gong, J. Wang, and X. Zhang, "Effect of pulsed electric field with variable frequency on coalescence of drops in oil," RSC Advances, vol. 5, no. 40, pp. 31318-31323, 2015.

[43] Y. Z. Zhang, Y. H. Liu, and R. J. Ji, "Dehydration efficiency of high-frequency pulsed DC electrical fields on water-inoil emulsion," Colloids and Surfaces A: Physicochemical and Engineering Aspects, vol. 373, no. 1-3, pp. 130-137, 2011.

[44] H. F. Gong and Y. Peng, "Effect of oil viscidity on optimal demulsion frequency in pulse electric field," Acta Petrologica Sinica, vol. 29, no. 1, pp. 168-173, 2013.

[45] H. F. Gong, Y. Peng, X. M. Zhang, Z. Yang, and H. Shang, "Effects of interfacial tension on deformation and vibration of droplet in emulsion oil under the action of pulse electric field," Acta Petrolei Sinica (Petroleum Processing Section), vol. 30, no. 6, pp. 1100-1105, 2014.

[46] H.-F. Gong, Z.-J. Yang, Y. Peng, and X.-M. Zhang, "Optimal parameters of high pulse electric field in oil demulsion," Journal of Chemical Engineering of Chinese Universities, vol. 29, no. 1, pp. 78-83, 2015.

[47] S. E. Taylor, "Theory and practice of electrically-enhanced phase separation of water-in-oil emulsions," Chemical Engineering Research and Design, vol. 74, no. 5, pp. 526-540, 1996.

[48] Q. R. Gan, "Study on motion behavior of liquid drop in the emulsion of high frequency pulse electric field," Oil and Gas Field Surface Engineering, vol. 25, pp. 5-6, 2006.

[49] G. Taylor, "Disintegration of water drops in an electric field," Proceedings of the Royal Society A: Mathematical, Physical and Engineering Sciences, vol. 280, no. 1382, pp. 383-397, 1964.

[50] T. Nishiwaki, K. Adachi, and T. Kotaka, "Deformation of viscous droplets in an electric field: poly (propylene oxide)/poly (dimethylsilone) systems," Langmuir, vol. 4, no. 1, pp. 170-175, 1988.

[51] H. F. Gong and Y. Peng, Dynamic Behavior and Mechanism of High Voltage Pulsed Electric Field, Chemical Industry Press, Beijing, China, 2015.

[52] H. Gong, Y. Tu, S. Song, and Y. Peng, "Steady deformation mechanics model of latex particle in emulsion under electric field," Acta Petrolei Sinica (Petroleum Processing Section), vol. 26, no. 4, pp. 600-604, 2010.

[53] D. H. Yang and L. M. He, "Factors influencing single drop deformation in high-voltage AC electric field," Journal of Chemical Industry and Engineering, vol. 62, pp. 1358-1364, 2011.

[54] O.-M. Midtgård, "Application of circuit theory for extraction of water from oil," International Journal of Circuit Theory and Applications, vol. 40, no. 9, pp. 927-942, 2012. 


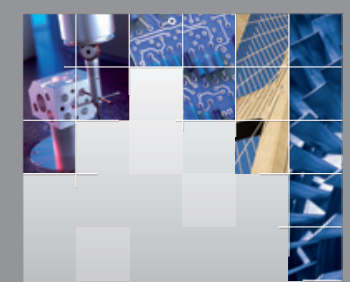

\section{Enfincering}
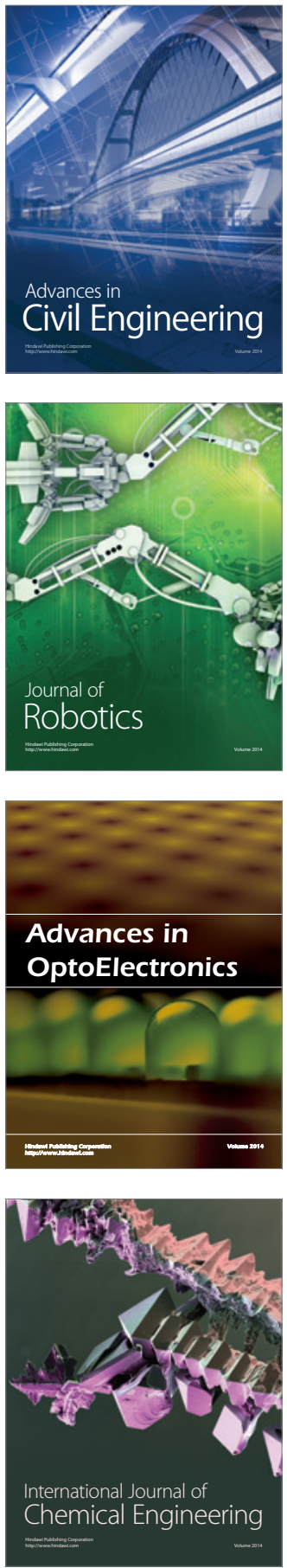

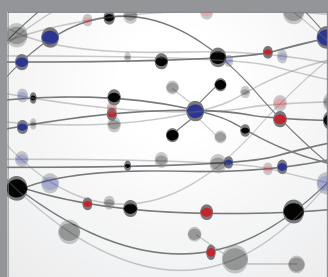

The Scientific World Journal

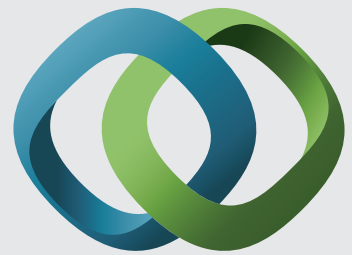

\section{Hindawi}

Submit your manuscripts at

http://www.hindawi.com
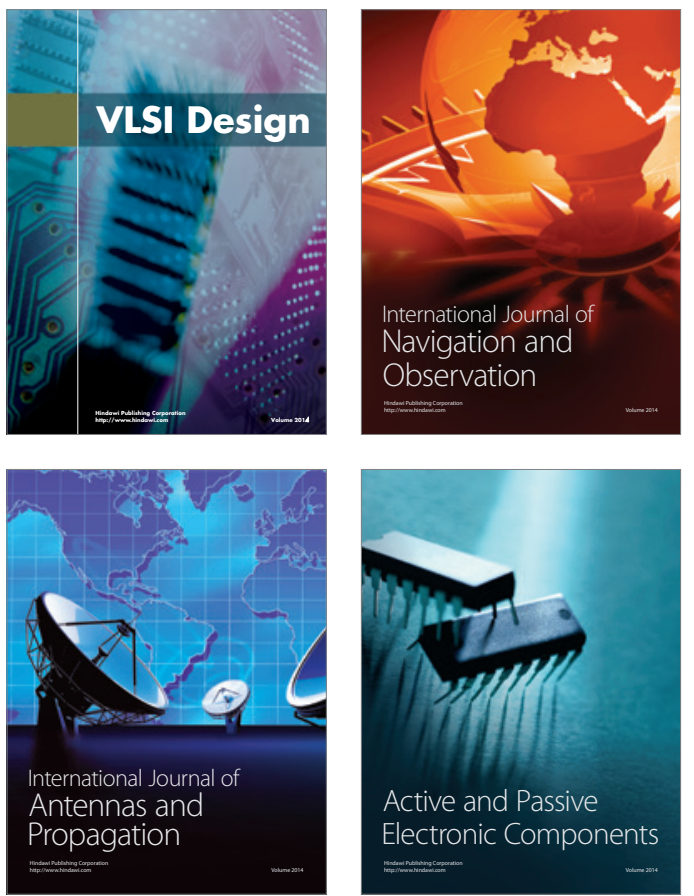
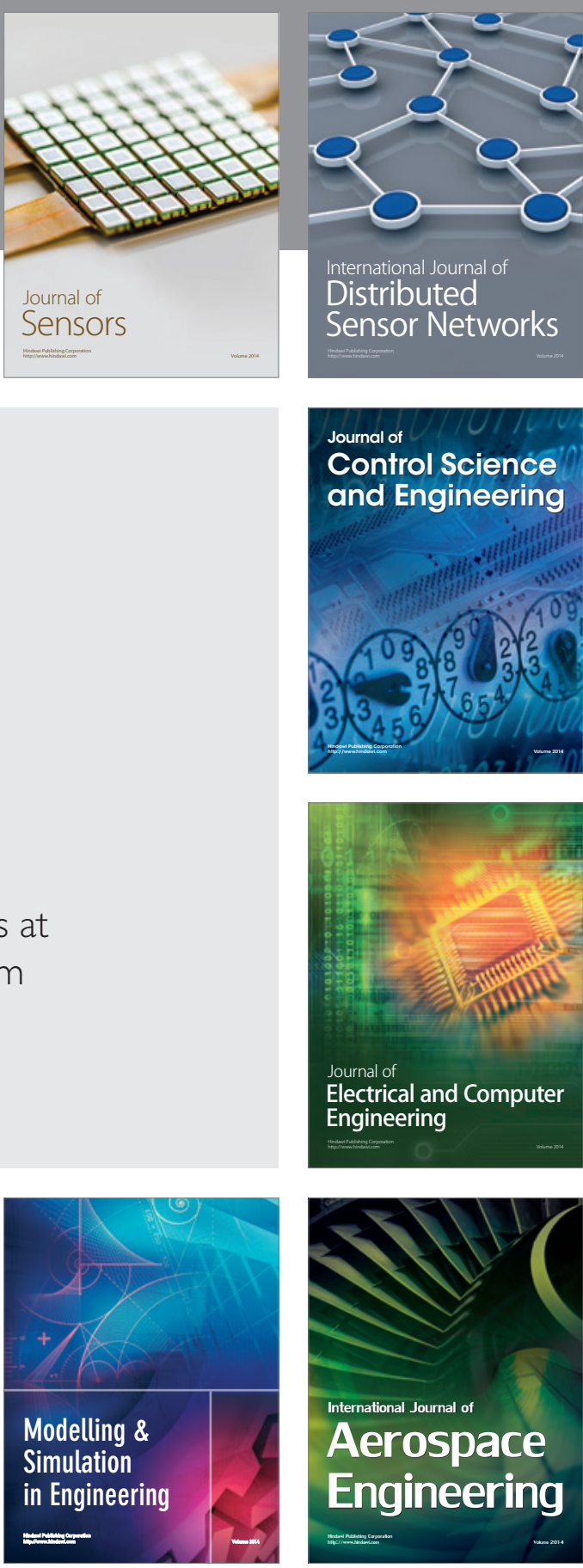

International Journal of

Distributed

Sensor Networks

Journal of

Control Science

and Engineering
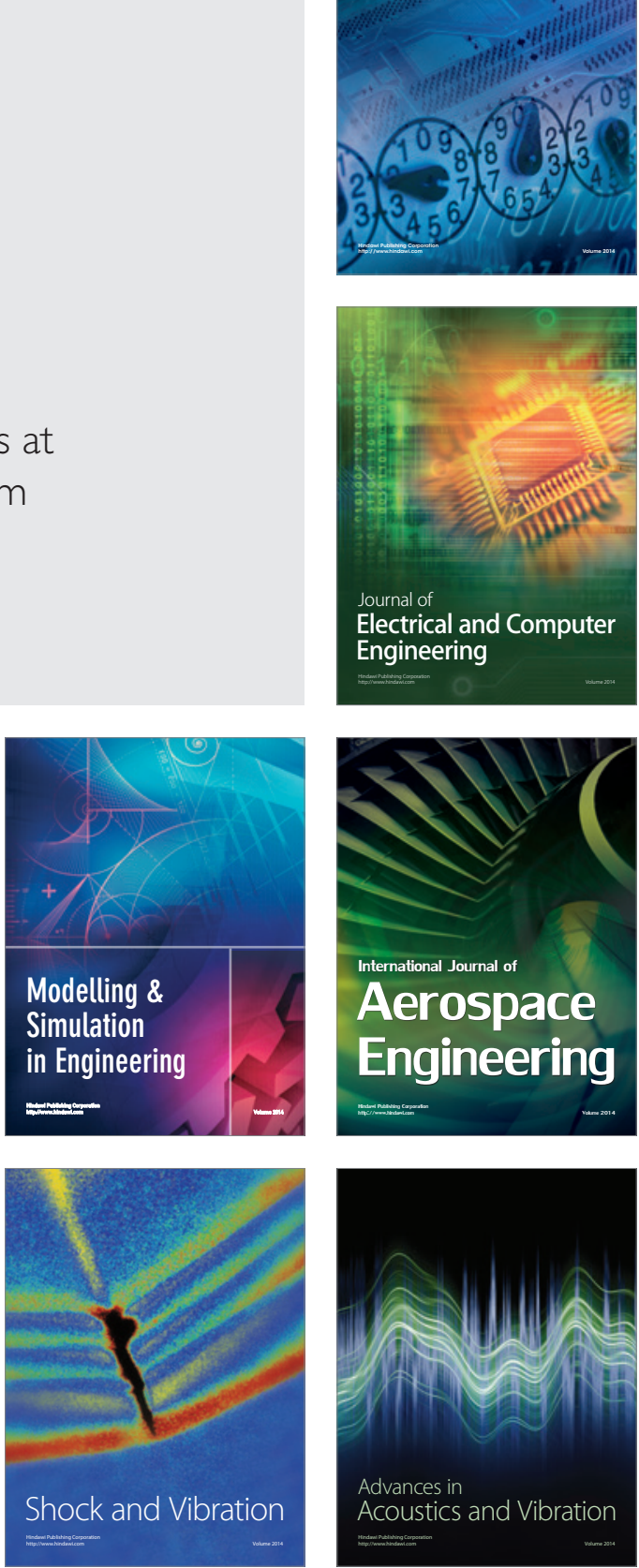